\title{
Protective factors for cognitive decline: Trajectories and changes in a longitudinal study with Italian elderly
}

Sara Mondini ( $\nabla$ sara.mondini@unipd.it)

Dept FISPPA, University of Padua

Veronica Pucci

Dept FISPPA, University of Padua

\section{Sonia Montemurro}

IRCCS, San Camillo Hospital, Venice

\section{Raffaella Rumiati}

International School for Advanced Studies

\section{Research Article}

Keywords: cognitive decline, cognitive reserve, dementia, education, occupation

Posted Date: April 28th, 2021

DOI: https://doi.org/10.21203/rs.3.rs-452796/v1

License: (c) (i) This work is licensed under a Creative Commons Attribution 4.0 International License. Read Full License 


\section{Abstract}

This retrospective study quantifies the relative contribution of age and sex as demographic factors, comorbidity, education and occupation as cognitive reserve (CR) proxies in accounting for cognitive aging. Participants underwent three neuropsychological evaluations, with their cognitive profiles being classified as unimpaired, mildly or severely impaired. For all 3,081 participants, in addition to age, education was the best predictor of performance followed by occupation. From the original sample, 543 participants were reassessed a second time, and 125 a third time. Depending on whether they maintained or worsened their profile based on their initial performance, participants were classified as resistant or declining. Results showed that, across assessments, the resistant had higher levels of education and occupation than the declining. Education and occupation modulated cognitive performance also in the more severely impaired group. This study confirms that the trajectories of changes of performance in ageing are consistently predicted by high levels of CR.

\section{Introduction}

Cognitive reserve (CR) is a construct that has been operationalized using several proxies such as education, occupation, indexes of quality of life and physical activity. High values of such proxies have been found to have a positive influence on cognitive health and to significantly discriminate between healthy and pathological ageing. Education has been repeatedly associated with lower risk of developing dementia and with improved cognitive functioning ${ }^{1,2}$. Indeed, individual differences in cognitive decline have often been observed in association with educational level ${ }^{3}$, with highly educated patients maintaining their functioning longer. In line with previous longitudinal cohort studies ${ }^{4}$, a recent longitudinal study involving 28,417 individuals confirmed the association between education and cognitive performance at baseline and between education and delayed onset of cognitive decline ${ }^{1}$.

As mentioned above, other factors, such as occupation or leisure activities ${ }^{5-7}$, have been hypothesized to contribute to $\mathrm{CR}$ in addition to formal education. Social, mental, and physical activities have beneficial effects on cognition in the elderly and have a protective role against dementia (see a review of longitudinal studies published between 1996 and 2004 by Fratiglioni, Paillard-Borg, \& Winblad, $2004^{8}$ ). A satisfactory social life is considered of great importance in decreasing the risk of morbidity and death in old age ${ }^{9}$, as well as in dementia ${ }^{10}$. An active daily social life involves a series of tasks that rely on cognition, and indeed older adults who have taken part in leisure activities show a lower risk of dementia than those with lesser involvement ${ }^{11}$.

The cognitive processes required for job duties may also contribute to $\mathrm{CR}^{12}$. Early evidence suggested that occupation, together with education, plays a crucial role against the development of dementia ${ }^{13}$. This has been replicated in subsequent studies with large groups ${ }^{12,14}$. One critical aspect that has been poorly investigated in previous studies is when and how the different CR proxies have an effect. In fact, most studies adopted a cross-sectional approach ${ }^{15}$ which does not allow to clearly verify how cognitive profiles change over time.

In this retrospective study, we investigated the effect of demographic factors (age and sex), presence of comorbidities and CR proxies (education and occupation) on normal and pathological aging, and their persistence over time. In Study 1, 3,018 individuals underwent a first neuropsychological assessment (T1) for suspected pathological aging. We predicted that the younger the age and the higher the cognitive reserve proxies, the better the cognitive outcomes. In Study 2, 543 participants underwent a second (T2), and 125 a third (T3) neuropsychological assessment. In addition, to replicate the same pattern found in Study 1, we expected to observe a slower cognitive decline over time in participants with higher levels of CR. 


\section{Methods And Results}

\section{1 Study 1}

From a cohort of 4,638 individuals, 3,081 (2,010 females) entered the study (mean age $=78.2 \mathrm{yrs}, \mathrm{SD}=7.79$, range $=45$ 99 ; mean education $=6.99 \mathrm{yrs}, \mathrm{SD}=3.85$, range $=0-25 \mathrm{yrs}$ ). Predictors of cognitive outcomes were age and sex as sociodemographic variables, comorbidity (number of concomitant relevant pathologies) as the clinical variable, and education and occupation as proxies of CR. Occupation was classified using the ISCO-08 code (International Standard Classification of Occupations, $\left.2008^{16}\right)$.

At each assessment, participants underwent a complete neuropsychological evaluation, but only six tests routinely used in our clinical unit, and known to be sensitive in detecting cognitive decline in the elderly, were selected for the present analysis: 1) MMSE (Mini-Mental State Examination17, Italian version18) for a global measure of cognition; 2) Famous Face naming test to measure proper name retrieval19; 3) Memory test, to measure episodic memory; 4) Fluency test, to measure lexical access and executive function; 5) Trail Making Test-A (TMT), to assess selective and spatial attention and psychomotor speed; and 6) Clock test to measure visuo-spatial abilities, planning and praxis. The last three tests are all taken from the ENB-2 battery20. Three diagnostic groups were identified based on psychometric data and anamnestic and clinical information: 1) Subjective Cognitive Decline21 (SCD) with 507 participants (17\%); 2) Mild Neurocognitive Disorder (MildND) with 584 participants (19\%); and 3) Major Neurocognitive Disorder due to Alzheimer's disease (MajorND) with 1980 participants (64\%).

All patients gave their written informed consent for the use of their data for research purposes at the moment of their consultation. The study was conducted in accordance with the Declaration of Helsinki, and it was approved by the Ethical Committee of the School of Psychology of the University of Padua.

\section{Results}

Generalized Linear Model (GLM) analyses were carried out to verify whether and how age, sex, education, occupation and comorbidities could predict participants' cognitive performance in the six tests. Models with best fit (after entering one predictor at time, based on R-squared) were those with all five predictors and all these models were significant (see Model fit measure in Table 1). A consistent effect of age, education, and occupation was found on all tests except for Clock and TMT where occupation had no effect. Sex and comorbidity played a minor role and predicted only two tests each.

Some differences among the three diagnostic groups (SCD, MildND, MajorND) were found, but, importantly, age and education predicted performance in all tests for each of the three groups. Occupation did not play a role within each groups, but it differs significantly among them $(F(2,900) 31.0, p<.001)$ : SCD had the lowest score (i.e., more complex jobs), while MajorND had the highest score (i.e., less complex jobs) (see Figure 1).

\subsection{Study 2}

Five hundred and forty-three individuals from Study 1 underwent a second assessment (T2), and 125 underwent a third assessment (T3). In a series of GLM analyses, age, sex, education, occupation and comorbidity were introduced as predictors, and participants' performance in the six cognitive tests as the dependent variable. At T2 all GLM were significant in predicting the outcomes in the cognitive tests. Education confirmed its effect on all six tests, and age had an effect only on two. No other effect was found. At T3, education was the only significant predictor in three tests.

Participants were then sorted into two groups based on clinical evidence and on their cognitive performance on the tests. At T2 and T3, individuals who performed worse than in the previous assessment, were put in a single group and named 
declining. This group also included MajorND participants who maintained the same clinical diagnosis but worsened their cognitive performance (no MajorND patient improved on cognitive tests). Participants who at T2 (or T3) did not worsen their profile were put in a single group and named resistant (see Supplementary Table 1 for more details about participants' changing cognitive profiles). At each assessment, the declining group showed lower average scores at T2 than at T1 and at T3 than at T2, on 4 out of 6 tests. Instead, the average scores on all six tests of the resistant did not change at either assessment T2 or T3 (see Table 2).

\section{Results}

The time-interval between assessments (from T1 to T2) was different among participants: Short (1 year; 242 participants), Medium (2-3 years; 207 participants) and Long (more than 3 years; 94 participants). Assuming that the longer the time, the higher the possible cognitive decline, the aim of this analysis was to verify if the effect of CR proxies changed depending on the time elapsed between assessments.

At T2, the resistant had higher education than the declining $(\mathrm{t}=8.04, \mathrm{df}=1064$, mean difference $=2.43, p<.001)$ and this difference was present at all time-intervals: Short $(\mathrm{t}=5.67, \mathrm{df}=480$, mean difference $=2.77, p<.001)$, Medium ( $\mathrm{t}=$ $3.02, \mathrm{df}=398$, mean difference $=1.34, p=.003)$, Long $(\mathrm{t}=5.43 \mathrm{df}=182$, mean difference $=3.77, p<.001)$.

Occupation showed the same trend as education: the resistant had higher occupations than the declining $(\mathrm{t}=-4.80, \mathrm{df}=$ 924 , mean difference $=-979, p<.001)$ across the three time-intervals: Short $(\mathrm{t}=-2.69, \mathrm{df}=424$, mean difference $=-819, p$ $=.007)$; Medium $(\mathrm{t}=-2.60, \mathrm{df}=342$, mean difference $=-858, p$-value $=.01)$; Long $(\mathrm{t}=-3.30, \mathrm{df}=154$, mean difference $=$ $-1565, p=.001)$. Note that the resistant had the highest education and more complex occupations when the interval was the longest (see Figure 2).

Of the 125 who underwent a third assessment (T3) 104 participants were declining (83.9\%) and only 21 (16.1\%) were resistant. Once again, resistant were more educated $(\mathrm{t}=3.86, \mathrm{df}=361$, mean difference $=2.49, p<.001)$ and with better occupation than declining $(\mathrm{t}=2.25, \mathrm{df}=319$, mean difference $=-941, p=0.025)$, (see Figure 3 ). The time-intervals among assessments were not considered in this analysis.

\section{Discussion}

In two studies, we aimed at identifying which factors may have a modulating effect on cognition in aging and how such an effect may change across time. In Study 1, age was found to be the best predictor of cognitive performance, consistently with the notion that cognition is known to deteriorate with aging. More importantly, education predicted participants' performance in all tests: the higher their education, the better their performance. Importantly, occupation was a good predictor of participants' performance in four out of six tests. The younger participants, the more educated ones, and those with more complex jobs showed better cognitive performance. While it is known that cognition deteriorates with aging, the effects shown by education and occupation support and strengthen the Cognitive Reserve hypothesis. Education as a protective factor has already received some attention ${ }^{3,22}$, but our findings clearly point to the relevance of occupation in operationalizing CR. Differently from education, which is mostly although not exclusively acquired when young, occupation is built on activities carried out during adulthood and demonstrates the benefits of lifelong learning mechanisms. Adult learning seems to be quite effective in preserving cognition in the elderly ${ }^{15,23}$.

Participants' gender made a difference only in two tests: females outperformed males in naming faces, while males outperformed females in the Clock. A similar verbal/visuo-spatial gender difference was observed in a previous study on the effect of CR on aging and found this verbal/visuo-spatial gender difference ${ }^{3}$, while Tappen et al. (2019) ${ }^{24}$ found that in an older adult population men were better than women at the Clock test. 
Age and education were confirmed as predictors of performance in all tests for all three diagnostic groups (SCD, MildND, and MajorND). Younger and with higher education individuals obtained better scores in each of the three groups. This finding highlights that education not only protects cognitively healthy people (i.e., SCD group), but it also protects MildND or MajorND patients from further decline. Interestingly, SCD were younger, more educated and held more complex jobs than the other two groups. Thus, it seems that less impaired individuals had CR proxies at the highest degree.

Across assessments, the explanatory power of predictors seems progressively attenuated. For instance, age was the best predictor of performance at T1, but at T2 it explained the performance only in two tests, and at T3 it showed no effect at all. The same trend was found for education, occupation and comorbidity. In fact, as age increases there is a multitude of factors which affects the elderly's cognition, and which gives rise to huge heterogeneity. Education was the only variable that continued to predict performance over time, although its effect decreased at T2 and T3.

Progressive worsening was found from one assessment to the next in most tests when considering all participants. However, when they were sorted into resistant (i.e., those who maintained their profile) or declining (i.e, those who worsened their profile), the former showed significantly higher education and more complex occupations than the latter at both T2 and T3. This result suggests a critical role of CR proxies in characterizing the evolution of dementia.

To our knowledge, this study is one of the few to include the category of Subjective Cognitive Decline individuals when $\mathrm{CR}$ is used as a potential modulator of age-related trajectories. Moreover, our findings are in line with the literature supporting a positive association between CR and cognitive performance in SCD individuals ${ }^{25,26}$.

In conclusion, our longitudinal study on healthy and pathological participants maintains that, together with social connectedness, ongoing sense of purpose, and ability to function independently, higher levels of cognitive reserve contribute to mental health and general wellbeing along the trajectories of aging.

\section{Data Availability}

The datasets generated during and/or analyzed during the current study are not publicly available due to information that could compromise participant privacy but are available from the corresponding author on reasonable request.

\section{References}

1. Clouston, S. A. P. et al. Education and Cognitive Decline: An Integrative Analysis of Global Longitudinal Studies of Cognitive Aging. J. Gerontol. B. Psychol. Sci. Soc. Sci. 75, e151-e160 (2020).

2. Stern, Y. et al. Whitepaper: Defining and investigating cognitive reserve, brain reserve, and brain maintenance. Alzheimer's Dement. 1305-1311 https://doi.org/10.1016/j.jalz.2018.07.219 (2020).

3. Le Carret, N. et al. The effect of education on cognitive performances and its implication for the constitution of the cognitive reserve. Developmental Neuropsychology. https://doi.org/10.1207/S15326942DN2303_1 (2003).

4. Zahodne, L. B. et al. Is residual memory variance a valid method for quantifying cognitive reserve? A longitudinal application. Neuropsychologia. 77, 260-266 (2015).

5. Stern, Y. et al. Relationship between lifetime occupation and parietal flow: implications for a reserve against Alzheimer's disease pathology.Neurology(1995).

6. Pettigrew, C. et al. Self-reported lifestyle activities in relation to longitudinal cognitive trajectories. Alzheimer Dis. Assoc. Disord. 33, 21-28 (2019). 
7. Rusmaully, J. et al. Contribution of cognitive performance and cognitive decline to associations between socioeconomic factors and dementia: A cohort study. PLoS Med. 14, 1-17 (2017).

8. Fratiglioni, L., Paillard-Borg, S. \& Winblad, B. An active and socially integrated lifestyle in late life might protect against dementia. Lancet Neurol. 3, 343-353 (2004).

9. Iwasaki, M. et al. Social networks and mortality based on the Komo-Ise cohort study in Japan. Int. J. Epidemiol. 31, 1208-1218 (2002).

10. Balouch, S., Rifaat, E., Chen, H. L. \& Tabet, N. Social networks and loneliness in people with Alzheimer's dementia. Int. J. Geriatr. Psychiatry. 34, 666-673 (2019).

11. Seeman, T. E. \& Crimmins, E. Social environment effects on health and aging: integrating epidemiologic and demographic approaches and perspectives. Ann. N. Y. Acad. Sci. 954, 88-117 (2001).

12. Pool, L. R. et al. Occupational cognitive requirements and late-life cognitive aging. Neurology. 86, 1386-1392 (2016).

13. White, L. et al. Association of education with incidence of cognitive impairment in three established populations for epidemiologic studies of the elderly. J. Clin. Epidemiol. 47, 363-374 (1994).

14. Andel, R. et al. Complexity of Work and Incident Cognitive Impairment in Puerto Rican Older Adults. J. Gerontol. B. Psychol. Sci. Soc. Sci. 74, 785-795 (2019).

15. Clare, L. et al. Potentially modifiable lifestyle factors, cognitive reserve, and cognitive function in later life: A crosssectional study. PLoS Med. 14, 1-14 (2017).

16. ILO. International Standard Classification of Occupations 2008 (ISCO-08): Structure, group definitions and correspondence tables. (2012).

17. Folstein, M. F., Folstein, S. E. \& McHugh, P. R. "Mini-mental state”: A practical method for grading the cognitive state of patients for the clinician. J. Psychiatr. Res. 12, 189-198 (1975).

18. Magni, E., Binetti, G., Bianchetti, A., Rozzini, R. \& Trabucchi, M. Mini-mental state examination: A normative study in Italian elderly population. European Journal of Neurology. 3, 198-202 (1996).

19. Semenza, C., Mondini, S., Borgo, F., Pasini, M. \& Sgaramella, M. T. Proper Names in Patients With Early Alzheimer's Disease. Neurocase. 9, 63-69 (2003).

20. Mondini, S., Mapelli, D., Vestri, A., Arcara, G. \& Bisiacchi, P. Esame neuropsicologico breve 2(2011).

21. Jessen, F. et al. A conceptual framework for research on subjective cognitive decline in preclinical Alzheimer's disease. Alzheimers. Dement. 10, 844-852 (2014).

22. Chan, D. et al. Lifestyle activities in mid-life contribute to cognitive reserve in late-life, independent of education, occupation, and late-life activities. Neurobiol. Aging. 70, 180-183 (2018).

23. VALENZUELA, M. J. \& SACHDEV, P. Brain reserve and dementia: a systematic review. Psychol. Med. 36, 441-454 (2006).

24. Tappen, R. The Clock Drawing Test. 12, 2-4 (2019).

25. Bessi, V. et al. From Subjective Cognitive Decline to Alzheimer's Disease: The Predictive Role of Neuropsychological Assessment, Personality Traits, and Cognitive Reserve. A 7-Year Follow-Up Study. J. Alzheimer's Dis. 63, 1523-1535 (2018).

26. Lojo-Seoane, C., Facal, D., Guàrdia-Olmos, J. \& Pereiro, A. X. \& Juncos-Rabadán, O. Effects of Cognitive Reserve on Cognitive Performance in a Follow-Up Study in Older Adults With Subjective Cognitive Complaints. The Role of Working Memory. Frontiers in Aging Neuroscience. 10, 189 (2018).

\section{Tables}




\begin{tabular}{|c|c|c|c|c|c|c|c|c|c|c|c|c|}
\hline \multirow[b]{3}{*}{ Models } & \multicolumn{12}{|c|}{ Predictors (Beta and $p$ ) } \\
\hline & \multicolumn{2}{|l|}{ Age } & \multicolumn{2}{|l|}{ Sex } & \multicolumn{2}{|c|}{ Education } & \multicolumn{2}{|c|}{ Occupation } & \multicolumn{2}{|c|}{ Comorb } & \multicolumn{2}{|c|}{$\begin{array}{l}\text { Model fit } \\
\text { measure }\end{array}$} \\
\hline & Beta & $p$ & Beta & $p$ & Beta & $p$ & Beta & $p$ & Beta & $p$ & $\mathrm{R}^{2}$ & $p$ \\
\hline MMSE & -0.250 & $<.001$ & 0.030 & .135 & 0.259 & $<.001$ & -0.048 & .037 & -0.030 & .111 & .164 & $<.001$ \\
\hline Memory & -0.312 & $<.001$ & 0.003 & .952 & 0.246 & $<.001$ & -0.067 & .017 & -0.020 & .367 & .201 & $<.001$ \\
\hline Faces & -0.300 & $<.001$ & -0.140 & .013 & 0.212 & $<.001$ & -0.066 & .042 & -0.039 & .141 & .175 & $<.001$ \\
\hline Fluency & -0.213 & $<.001$ & 0.021 & .065 & 0.268 & $<.001$ & -0.067 & .016 & -0.077 & $<.001$ & .166 & $<.001$ \\
\hline TMT & 0.250 & $<.001$ & -0.035 & .497 & -0.271 & $<.001$ & 0.052 & .083 & 0.083 & $<.001$ & .192 & $<.001$ \\
\hline Clock & -0.193 & $<.001$ & 0.187 & $<.001$ & 0.247 & $<.001$ & -0.017 & .568 & -0.006 & .803 & .134 & $<.001$ \\
\hline
\end{tabular}

Table 1. GLM significance for each test. The Table shows the contribution of each predictor to the model. Model fit measures indicate the significance of models with all covaried predictors.

\begin{tabular}{|c|c|c|c|c|c|c|}
\hline & \multicolumn{6}{|l|}{ declining } \\
\hline & \multicolumn{3}{|c|}{ From T1 to $\mathrm{T} 2$} & \multicolumn{3}{|c|}{ From T2 to T3 } \\
\hline & $t(d f)$ & mean diff. & $p$-value & $t(d f)$ & mean diff. & $p$-value \\
\hline MMSE & 11.4 (399) & 2.80 & $<.001$ & $5.92(92)$ & 2.47 & $<.001$ \\
\hline Memory & $4.15(220)$ & 0.64 & .005 & $5.35(51)$ & 4.75 & $<.001$ \\
\hline Faces & $3.92(83)$ & 0.99 & $<.001$ & 3.17 (17) & 1.33 & .006 \\
\hline Fluency & 1.18 (176) & 0.24 & .240 & $1.62(52)$ & 0.63 & .111 \\
\hline TMT & $-3.08(182)$ & -14.25 & .002 & $-1.97(54)$ & -15.38 & .054 \\
\hline \multirow[t]{4}{*}{ Clock } & $1.75(225)$ & 0.37 & .082 & $2.26(64)$ & 0.79 & .027 \\
\hline & \multicolumn{6}{|l|}{ resistant } \\
\hline & \multicolumn{3}{|c|}{ From $\mathrm{T} 1$ to $\mathrm{T} 2$} & \multicolumn{3}{|c|}{ From T2 to T3 } \\
\hline & $t(d f)$ & mean diff. & $p$-value & $t(d f)$ & mean diff. & $p$ \\
\hline MMSE & $0.32(81)$ & 0.11 & .710 & $1.20(15)$ & 1.00 & .248 \\
\hline Memory & $1.29(87)$ & 1.06 & .200 & $0.87(17)$ & 1.28 & .398 \\
\hline Faces & $1.58(29)$ & 0.73 & .125 & $2.61(3)$ & 1.25 & .080 \\
\hline Fluency & $0.32(83)$ & 0.13 & .751 & $-1.99(17)$ & -1.52 & .066 \\
\hline TMT & $-0.42(29)$ & -1.56 & .672 & $-1.46(15)$ & -6.63 & .165 \\
\hline Clock & $-1.04(60)$ & -0.34 & .301 & 0-38 (13) & 0.18 & .707 \\
\hline
\end{tabular}

Table 2. Resistant and declining. Paired sample T-tests which show the mean difference of the 6 tests between T1 and T2 and between T2 and T3 in the two groups separately (declining and resistant). 


\section{Figures}

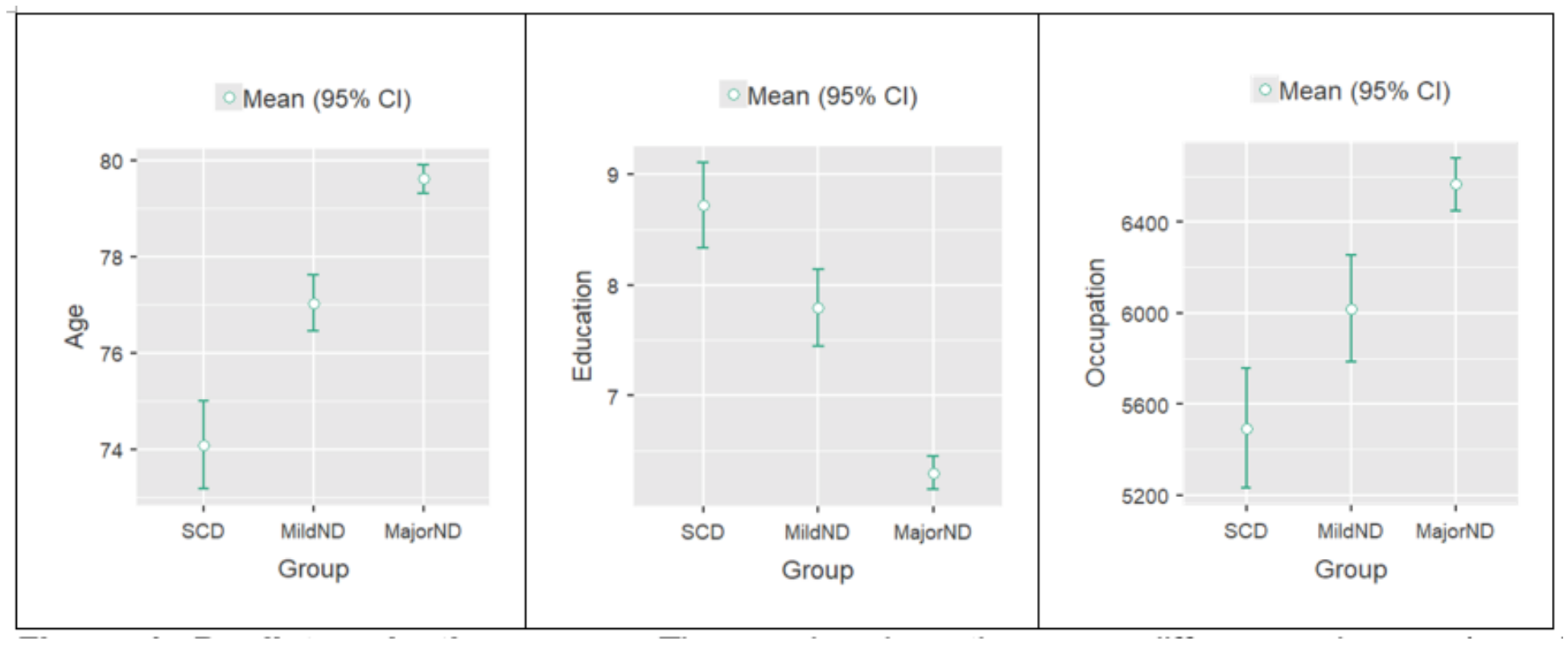

Figure 1

Predictors in the groups. The graphs show the mean differences in age (years), education (years) and occupation (ISCO08 codes: lower scores indicate more complex occupations) resulting from one-way ANOVA among the three groups (SCD, MildND and MajorND). 


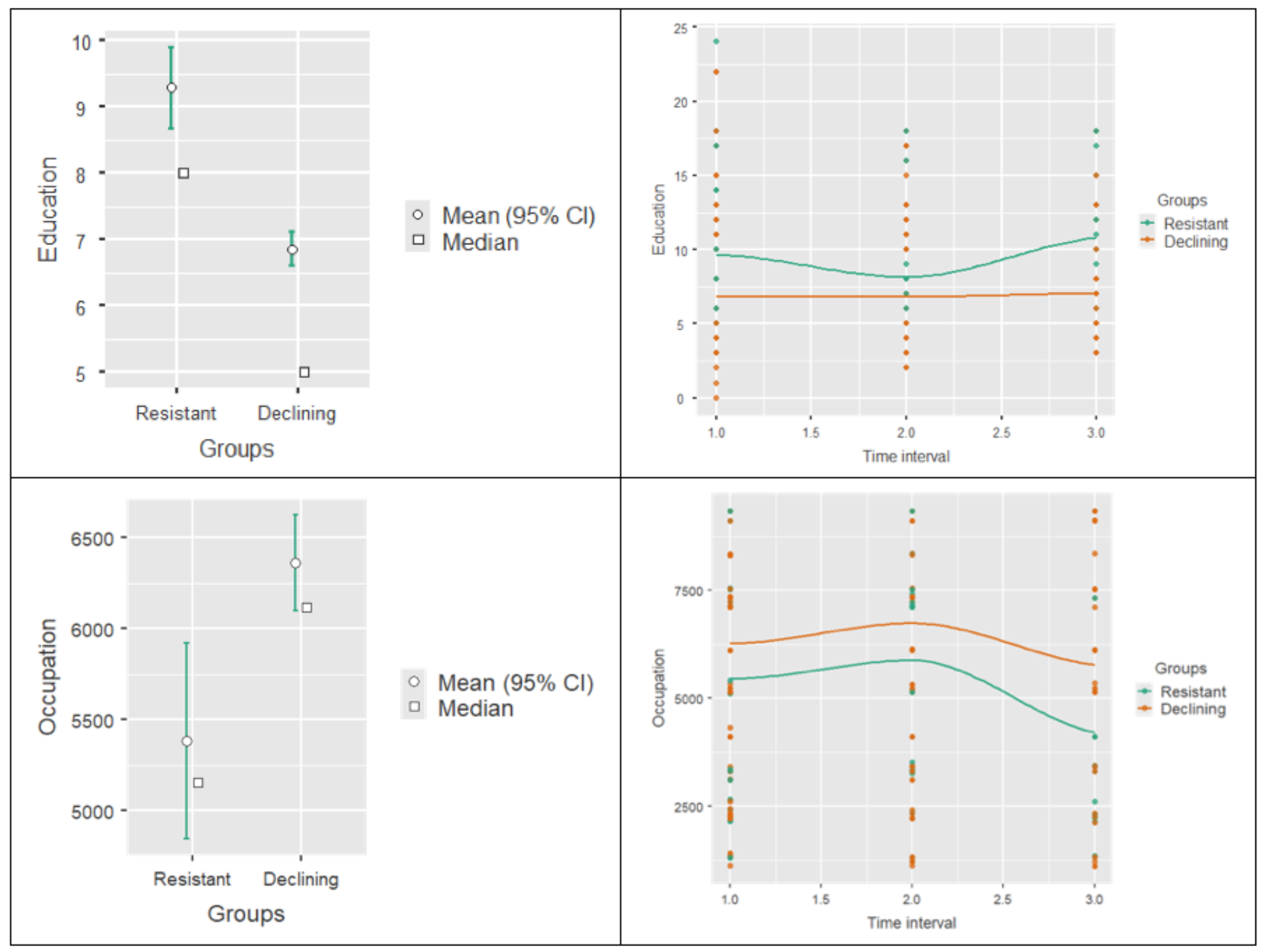

Figure 2

Education and occupation effects on resistant and declining individuals at the second assessment, on different time intervals. The two graphs on the left-hand side show the mean difference of education and occupation between resistant and declining, while the two graphs on the right-hand side show the mean difference of education and occupation between resistant and declining on the three time intervals. 


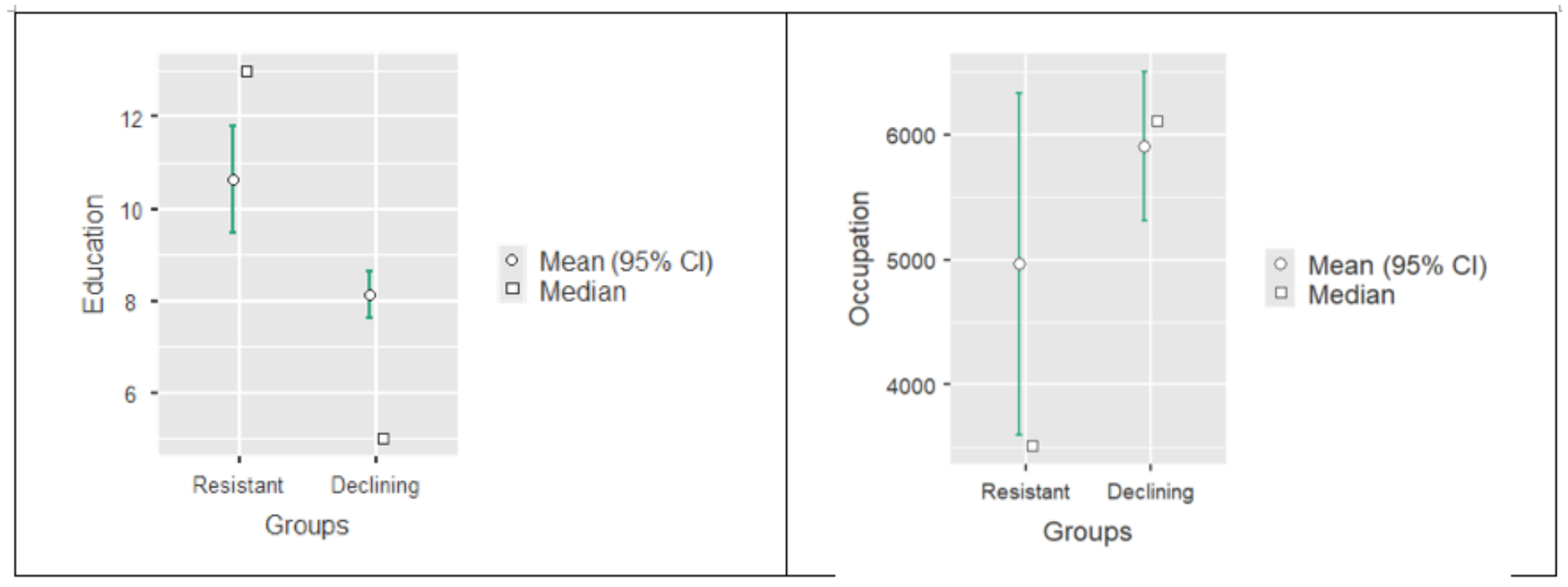

\section{Figure 3}

Education and occupation effects on resistant and declining individuals at the third assessment. The graphs represent the mean difference of education (on the left-hand side) and occupation (on the right-hand side) between resistant and declining at the third assessment (T3).

\section{Supplementary Files}

This is a list of supplementary files associated with this preprint. Click to download.

- Supplementary.pdf 